\title{
Terahertz Bessel Beam Applied to Thickness Measurement of Ellipsometry Methods
}

\author{
Siyu Tu, Kejia Wang*, Jinsong Liu, Zhengang Yang \\ Wuhan National Laboratory for Optoelectronics, Huazhong University of Science and Technology, Wuhan, China \\ Email: ${ }^{\star 764184066 @ q q . c o m ~}$
}

How to cite this paper: Tu, S.Y., Wang, K.J., Liu, J.S. and Yang, Z.G. (2021) Terahertz Bessel Beam Applied to Thickness Measurement of Ellipsometry Methods. Journal of Computer and Communications, 9, 125-132. https://doi.org/10.4236/jcc.2021.912010

Received: August 28, 2021

Accepted: December 28, 2021

Published: December 31, 2021

\begin{abstract}
This paper introduces a Terahertz (THz) ellipsometer thickness measurement method based on Bessel beams. The ellipsometry method is used to measure the thickness of film in the $\mathrm{THz}$ band. And the thickness of film could be measured in the range of hundreds of microns which the measurement of film thickness by ellipsometer is usually only a few hundred $\mathrm{nm}$ in visible light. In addition, the photon energy of the THz-wave is very low and has little damage to the film. The $\mathrm{THz}$ Bessel beam has the characteristics of self-healing and diffraction-free. It can make the film thickness measurement within the diffraction-free distance, which is conducive to the flexibility of the ellipsometer system. We use a multi-frequency method to measure film with two-dimensional finite different time domain (FDTD) to numerical simulation analysis of light intensity successfully.
\end{abstract}

\section{Keywords}

Bessel-Beam, Ellipsometry, Diffraction-Free, $\mathrm{THz}$

\section{Introduction}

Terahertz $(\mathrm{THz})$ waves are electromagnetic waves with a frequency from 0.1 $\mathrm{THz}$ to $10 \mathrm{THz}$ and a wavelength from $30 \mathrm{um}$ to $3 \mathrm{~mm}$, which are between microwaves and infrared waves. $\mathrm{THz}$ waves have the characteristics of both microwaves and infrared waves. Macroscopically, $\mathrm{THz}$ waves can penetrate many dielectric materials and non-polar liquids. Microscopically, $\mathrm{THz}$ waves contain rich sample spectral information due to their broad spectrum [1] [2]. The unique characteristics of $\mathrm{THz}$ waves have brought far-reaching influence on communication (broadband communication), radar, electronic countermeasures, electromagnetic weapons, astronomy, medical imaging, non-destructive testing, safety inspection [3]-[8]. 
With thin film technology in information storage, electronic components, aerospace technology and optical instruments, etc. The accurate measurement of optical properties and surface morphology of film has gradually become an important direction of film research. Moreover, the physical properties of the film are closely related to the optical properties and thickness of the film. Therefore, the precise measurement of the optical properties and thickness of the film has become the primary research issue. The current methods for measuring the refractive index and thickness of the film include ellipsometry, heterodyne interferometry, equal thickness interferometry, reflection spectroscopy, transmission spectroscopy and other measurement methods [9] [10]. Ellipsometry is a non-contact and non-destructive measurement, which can measure samples under vacuum, atmosphere, moisture, and harmful environments with low environmental requirements [11] [12]. In addition, the ellipsometry is simple to operate and has a short measurement period. It can measure multiple physical quantities (film thickness, film refractive and absorption coefficient) at the same time. Hence, ellipsometry is widely used to measure films.

The Bessel beam has diffraction-free characteristics, the intensity distribution remains unchanged during propagation and has a high degree of localization [13] [14] [15]. It is widely used in precision measurement, laser processing and other fields in the optical waveband. Th diffraction-free and long focal depth of Bessel beams could be further used in $\mathrm{THz}$ there-dimensional (3D) tomography systems, such as THz CT systems [16]. The THz imaging system based on Bessel beam has unique advantages in applications such as long-distance standing human security inspection and non-destructive inspection of thick sample.

The film thickness measured by ellipsometer in the optical band is mainly in the range of hundreds of nanometers. For samples with a thickness of millimeters and hundreds of microns, the visible light waveband cannot be measured. Therefore, we proposed the application of $\mathrm{THz}$ waves to accurately measure the thickness of samples in the order of millimeters and hundreds of microns. In addition, the photon energy of the $\mathrm{THz}$ wave is relatively low, which hardly cause damage to the sample. The THz Bessel beam has the characteristics of diffraction-free and self-healing, and can realize precise measurement of samples within its diffraction-free distance. Thus, an ellipsometer thickness measurement method based on $\mathrm{THz}$ Bessel beam is proposed in this paper. This paper is organized as follows. In Section 2, the theory of ellipsometry calculation of film thickness and the generation of $\mathrm{THz}$ Bessel beams are showed. In Section 3, numerical simulation calculation of the thickness of film measured by $\mathrm{THz}$ Bessel beam is carried out by two-dimensional finite difference time domain (FDTD). Finally, our conclusion is summarized in Section 4.

\section{Theory}

\subsection{The Generation of THz Bessel Beam}

A Gaussian beam with a waist radius of $\omega_{0}$ is normally incident on the front 
surface of the axicon [17]. By geometric optics analysis, the Gaussian beam is regarded as a straight line, in Figure 1. According to refraction law:

$$
\begin{aligned}
& n_{0} \sin (\theta)=n \sin (\gamma), \\
& \alpha_{0}+\gamma=\theta, \tau+2 \gamma=\pi,
\end{aligned}
$$

the refractive index of atmosphere is $n_{0}=1$ :

$$
\theta=\arcsin \left[\frac{n}{n_{0}} \cos (\tau / 2)\right]=\arcsin [n \cos (\tau / 2)],
$$

the angle between the Gaussian beam and the $\mathrm{z}$-axis after refractive:

$$
\alpha_{0}=\arcsin [n \cos (\tau / 2)]+(\tau-\pi) / 2,
$$

when $\gamma$ is small:

$$
\begin{gathered}
\theta=n \gamma, \\
\alpha_{0}=(n-1) \gamma .
\end{gathered}
$$

Therefore, the Bessel beam with a diffraction-free distance of $z_{\max }=\omega_{0} / \tan \left(\alpha_{0}\right)$ is generated.

\subsection{Ellipsometry Measurement Theory}

The basic principle of ellipsometer measurement is to incident a beam of elliptic polarization light onto the sample to be tested at a certain angle of incidence. Since the sample has different reflection and transmission coefficients for the p-light and s-light of the incident light, the polarization state of the light reflected from the sample changes with respect to the incident light which is showed in Figure 2. The variation of polarization state is closely related to the structure of sample. The specific structure of sample can be determined according to the variation of polarization state. The variation of the polarization state of the light before and after reflection is observed to determine the reflection coefficient ratio $\rho$, which the information related to the optical parameters of the sample could be obtained.

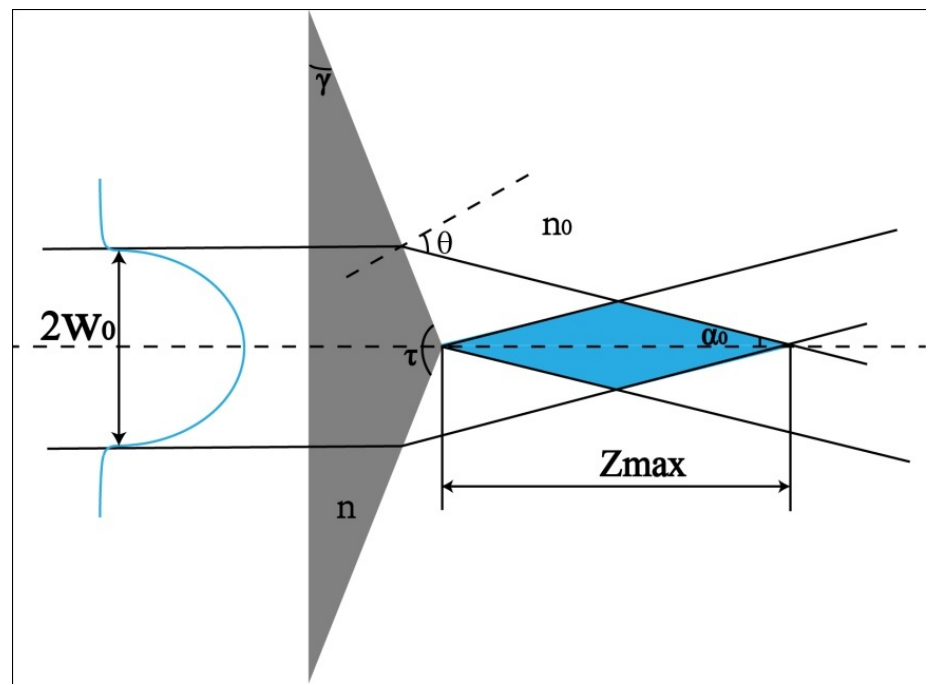

Figure 1. The theory of Bessel beam. 
The electric vector of incident light can be decomposed into p-light and s-light. $E_{i p}$ and $E_{i s}$ represents the p-light and s-light of the incident light respectively. $E_{r p}$ and $E_{r s}$ represents the p-light and s-light of the incident light respectively.

$$
\begin{aligned}
r_{p} & =E_{r p} / E_{i p} \\
r_{s} & =E_{r s} / E_{\text {is }}
\end{aligned}
$$

The Fresnel formula for the reflection of wave on the interface:

$$
\begin{gathered}
r_{p}=\frac{n_{2} \cos \varphi_{1}-n_{2} \cos \varphi_{2}}{n_{2} \cos \varphi_{1}+n_{1} \cos \varphi_{2}} \\
r_{s}=\frac{n_{1} \cos \varphi_{1}-n_{2} \cos \varphi_{2}}{n_{1} \cos \varphi_{1}+n_{2} \cos \varphi_{2}}
\end{gathered}
$$

The relationship between the complex amplitude of the total reflected wave and the incident wave is:

$$
\begin{gathered}
E_{r p}=\frac{r_{1 p}+r_{2 p} \exp (-i 2 \delta)}{1+r_{1 p} r_{2 p} \exp (-i 2 \delta)} E_{i p} \\
E_{r s}=\frac{r_{1 s}+r_{2 s} \exp (-i 2 \delta)}{1+r_{1 s} r_{2 s} \exp (-i 2 \delta)} E_{i s}
\end{gathered}
$$

The total reflection coefficients of the film are:

$$
\begin{aligned}
& R_{p}=E_{r p} / E_{i p} \\
& R_{p}=E_{r p} / E_{i p}
\end{aligned}
$$

The reflectance coefficient:

$$
\begin{aligned}
\rho & =R_{p} / R_{s}=\frac{E_{r p} / E_{i p}}{E_{r s} / E_{i s}}=\left|\frac{E_{r p} / E_{i p}}{E_{r s} / E_{i s}}\right| \exp \left\{i\left[\left(\beta_{r p}-\beta_{i p}\right)-\left(\beta_{r s}-\beta_{i s}\right)\right]\right\} \\
& =\tan \psi \exp (i \Delta)
\end{aligned}
$$

According to the Fresnel formula and the law of refraction:

$$
\left\{\begin{array}{l}
r_{1 p}=\left(n_{2} \cos \varphi_{1}-n_{1} \cos \varphi_{2}\right) /\left(n_{2} \cos \varphi_{1}+n_{1} \cos \varphi_{2}\right) \\
r_{2 p}=\left(n_{3} \cos \varphi_{2}-n_{2} \cos \varphi_{3}\right) /\left(n_{3} \cos \varphi_{2}+n_{2} \cos \varphi_{3}\right) \\
r_{1 s}=\left(n_{1} \cos \varphi_{1}-n_{2} \cos \varphi_{2}\right) /\left(n_{1} \cos \varphi_{1}+n_{2} \cos \varphi_{2}\right) \\
r_{2 s}=\left(n_{2} \cos \varphi_{2}-n_{3} \cos \varphi_{3}\right) /\left(n_{2} \cos \varphi_{2}+n_{3} \cos \varphi_{3}\right) \\
n_{1} \sin \varphi_{1}=n_{2} \sin \varphi_{2}=n_{3} \sin \varphi_{3} \\
2 \delta=\left(4 \pi d n_{2} \cos \varphi_{2}\right) / \lambda
\end{array}\right.
$$

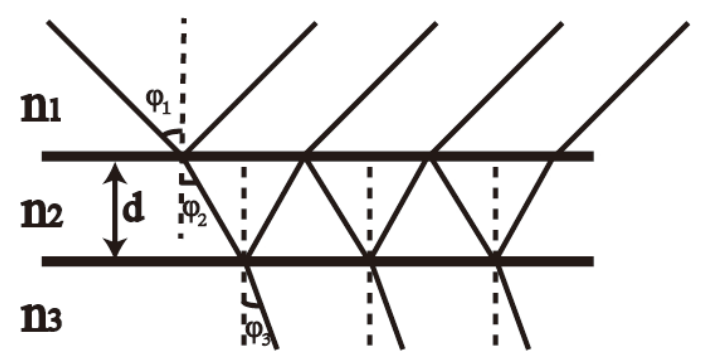

Figure 2. The film reflection. 
Therefore, the relevant parameters of the film can be obtained by the variation of the polarization state of the incident beam before and after reflection.

\section{Simulation Results}

The 2D FDTD (Finite Difference Time Domain) numerical stimulation method is utilized to conduct a simulation on the model. In Figure 3, a Gaussian beam with a waist radius of $20 \mathrm{~mm}$ is incident on an axicon with base angle of $15^{\circ}$. The Bessel beam is generated by the axicon. The sample is within the diffraction-free distance of the Bessel beam. And the Bessel beam is incident on the film obliquely at an incident angle of $45^{\circ}$. A line monitor is set in the reflection direction of the beam.

The basic setup of the model is as follows. Since the thickness of the measured film is related to the polarization state of the incident beam, the polarization state is specially set when setting Gaussian beam source. We choose to use circularly polarization beam as the incident light. In FDTD, a Gaussian beam source can only set a certain angle of linear polarization light. Therefore, we set up two Gaussian beam sources. The amplitudes of two sources are same. Their polarization angles are $0^{\circ}$ and $90^{\circ}$ respectively as the p-light and s-light. The phase difference between the two Gaussian beams is $90^{\circ}$. In Solidworks software, an isosceles triangle with a base angle of $15^{\circ}$ and a bottom margin of three inches is created. The isosceles triangle is rotated $360^{\circ}$ around its center line to form axicon. The axicon is exported from Solidworks in STL format and imported it to FDTD. A sample with film thickness of $1 \mathrm{~mm}$, refractive index $n=1.6$, substate thickness of $20 \mathrm{~mm}$, refractive index $n=3.4$ was established in FDTD. The position of the Gaussian sources, axicon, sample, monitor are respectively designed in the appropriate position.

In Figure 4, it is showed that the Gaussian beam is incident on the axicon to produce a Bessel beam. And the Bessel beam is incident on the film, part of the Bessel beam is reflected, part of the beam pass through the substrate. Figure 5 are the light intensity measured by the line monitor. It can be seen that the strong middle peak is the reflected beam of the main lobe of the Bessel beam. Figure 5(a) \& Figure 5(b) is the p-light and s-light of the Bessel beam generated by the axicon respectively, which is also the incident light. Figure 5(c) \& Figure 5(d) is

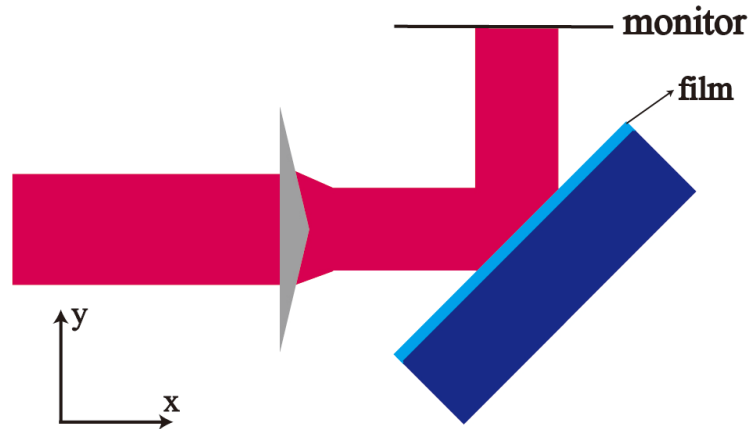

Figure 3. Simulation model. 


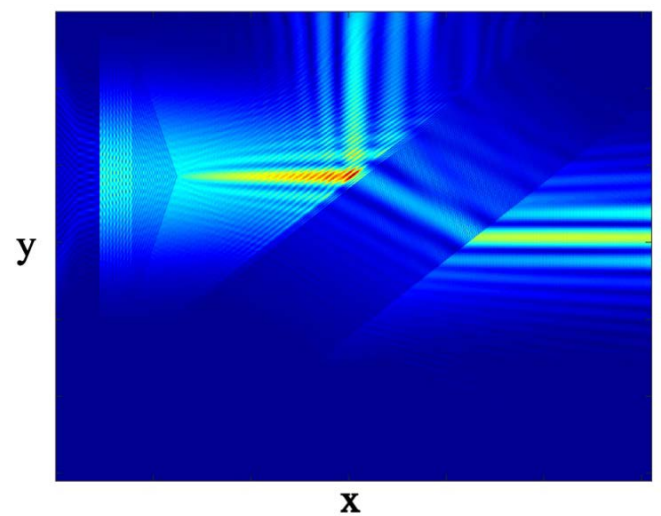

Figure 4. The electric field of xy cross-section.
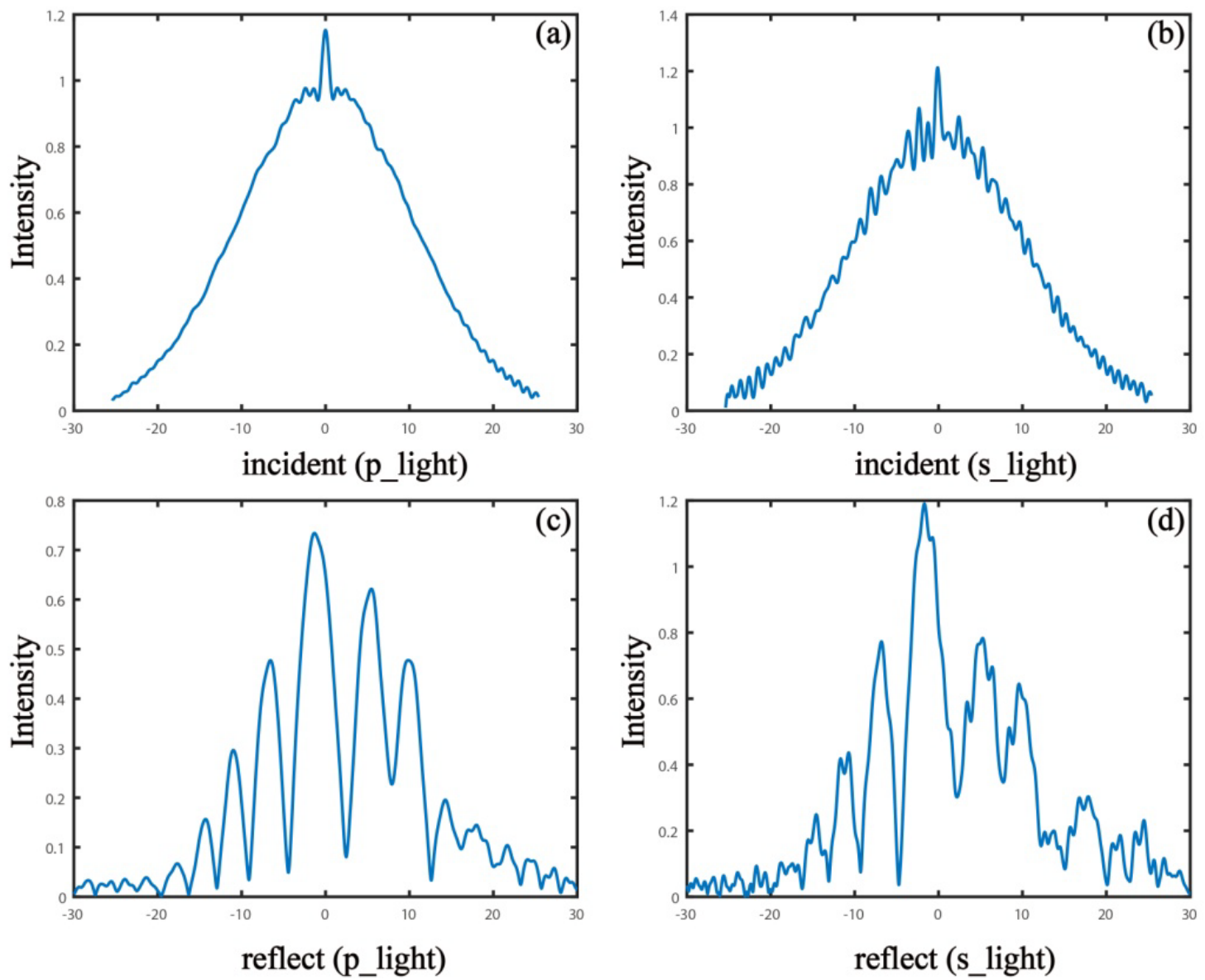

Figure 5. (a) The intensity of incident p-light, (b) The intensity of incident s-light, (c) The intensity of reflect p-light, (d) The intensity of reflect s-light.

the p-light and s-light reflected by film. The ellipsometric parameters can be calculated by the light intensity of the incident and reflected p-light and s-light of simulation. The p-light to s-light ratio of incident light is 0.9959 , the p-light to s-light of reflected light is 0.6524 . According to calculation, the $r_{1 p}$ is 0.1155 , the $r_{2 p}$ is 0.3218 , the $r_{1 s}$ is -0.3399 , the $r_{2 s}$ is -0.3971 , the ellipsometric parameters is $\tan \psi=0.655062567862263$. By: 


$$
2 \delta=\left(4 \pi d n_{2} \cos \varphi_{2}\right) / \lambda,
$$

the Periodic film thickness is obtained.

The Periodic film thickness value is as follows:

Table 1. Part of the periodic film thickness.

\begin{tabular}{|l|l|l|l|l|l|l|l|}
\hline$d / \mathrm{mm}$ & 0.7558 & 0.8322 & 1.0279 & 1.2897 & 1.5852 & 1.8987 & 2.2226 \\
\hline
\end{tabular}

Table 1 gives part of the Periodic film thickness. When setting up model in simulation, the film thickness is $1 \mathrm{~mm}$. Therefore, the value of the calculated periodic film thickness closest to $1 \mathrm{~mm}$ is the actual value of the film thickness. The $1.0279 \mathrm{~mm}$ is closest to $1 \mathrm{~mm}$, and there is only $0.02 \mathrm{~mm}$ error which proves that the Bessel beam model is correct.

\section{Conclusion}

This paper proposed a method of measuring film thickness by $\mathrm{THz}$ Bessel beam. It is proved that precise thickness measurement of thin films of millimeters and hundreds of microns can be achieved in the $\mathrm{THz}$ band. And the $\mathrm{THz}$ Bessel beam is a diffraction-free beam, which can realize the measurement system with a long depth of field. We have employed the ellipsometric calculation method in FDTD to verify the feasibility of this theory. In the future, we will further implement ellipsometric thickness measurement in the $\mathrm{THz}$ experimental system.

\section{Conflicts of Interest}

The authors declare no conflicts of interest regarding the publication of this paper.

\section{References}

[1] Redo-Sanchez, A.S., et al. (2016) Terahertz Time-Gated Spectral Imaging for Content Extraction through Layered Structures. Nat Commun, 7, 12665. https://doi.org/10.1038/ncomms12665

[2] Sun, W., Wang, X. and Zhang, Y. (2012) A Method to Monitor the Oil Pollution in Water with Reflective Pulsed Terahertz Tomography. Optik, 123, 1980-1984. https://doi.org/10.1016/j.ijleo.2011.10.002

[3] Hu, B.B. and Nuss, M.C. (1995) Imaging with Terahertz Waves. Optics Letters, 20, 1716. https://doi.org/10.1364/OL.20.001716

[4] Wu, Q., Hewitt, T.D. and Zhang, X.C. (1996) Two-Dimensional Electro-Optic Imaging of THz Beams. Applied Physics Letters, 69, 1026-1028. https://doi.org/10.1063/1.116920

[5] Lui, H.S., Taimre, T., Bertling, X., et al. (2014) Terahertz Inverse Synthetic Aperture Radar Imaging Using Self-Mixing Interferometry with a Quantum Cascade Laser. Optics Letters, 39, 2629-2632. https://doi.org/10.1364/OL.39.002629

[6] Song, H.J. and Nagatsuma, T. (2011) Present and Future of Terahertz Communications. IEEE Transactions on Terahertz Science and Technology, 1, 256-263. https://doi.org/10.1109/TTHZ.2011.2159552 
[7] Seeds, A.J., Shams, H., Fice, M.J., et al. (2015) Terahertz Photonics for Wireless Communications. Journal of Lightwave Technology, 33, 579-587. https://doi.org/10.1109/JLT.2014.2355137

[8] Shen, Y.C. (2007) Terahertz Pulsed Spectroscopy and Imaging for Pharmaceutical Applications: A Review. J Pharm Pharmacol, 59, 209.

[9] Paulick, T.C. (1986) Inversion of Normal-Incidence (R,T) Measurements to Obtain n+ik for Thin Films. Applied Optics, 25, 562-564. https://doi.org/10.1364/AO.25.000562

[10] Chiu, M., Lee, J. and Su, D. (1999) Complex Refractive-Index Measurement Based on Fresnel's Equations and the Use of Heterodyne Interferometry. Applied Optics, 38, 4047-4052. https://doi.org/10.1364/AO.38.004047

[11] Kravets, V.G., Grigorenko, A.N., et al. (2010) Spectroscopic Ellipsometry of Graphene and an Exciton-Shifted van Hove Peak in Absorption. Physical Review B, 81, 1-6. https://doi.org/10.1103/PhysRevB.81.155413

[12] Li, J., Ramanujam, B. and Collins, R.W. (2011) Dual Rotating Compensator Ellipsometry: Theory and Simulations. Thin Solid Films, 519, 2725-2729.

https://doi.org/10.1016/j.tsf.2010.11.075

[13] Cerda, S.C., Nava, M.M. and Hickmann, J.M. (1998) Interference of Traveling Nondiffracting Beams. Optics Letters, 23, 1871-1873. https://doi.org/10.1364/OL.23.001871

[14] Durnin, J., Miceli Jr., J. and Eberly, J. (1987) Diffraction-Free Beams. Physical Review Letters, 58, 1499. https://doi.org/10.1103/PhysRevLett.58.1499

[15] Scott, G. and Mcardle, N.J.O.E. (1992) Efficient Generation of Nearly Diffraction-Free Beams Using an Axicon. 31, 2640-2643. https://doi.org/10.1117/12.60017

[16] Bitman, A., Goldring, S., Moshe, I., et al. (2014) Computed Tomography Using Broadband Bessel THz Beams and Phase Contrast. Optics Letters, 39, 1925-1928. https://doi.org/10.1364/OL.39.001925

[17] Monk, S., Arlt, J., Robertson, D.A., et al. (1999) Generation of Bessel Beams at Millimeter Waves Frequencies by Use of an Axicon. Optics Communications, 170, 213-215. https://doi.org/10.1016/S0030-4018(99)00463-0 